\title{
Solution to Monthly Problem 11277
}

\author{
David H. Bailey* Jonathan M. Borwein ${ }^{\dagger}$
}

March 23, 2007

The Monthly problem \#11277 [1] asks to find

$$
\alpha=\int_{0}^{\pi / 2} \int_{0}^{\pi / 2} \frac{\log (1+\sin \theta \cos \phi) \sin \theta d \theta d \phi}{1+\sin ^{2} \theta \cos ^{2} \phi}
$$

When the first of the present authors saw this problem, he quickly evaluated its numerical value, using the "QD" software package (which performs arithmetic, together with 1-D, 2-D and 3-D numerical integration, to roughly 62 -digit precision). The numerical value obtained was:

$$
0.427568028991069784052967447122131004456236190511744594707758239
$$

After pasting in this numerical value to the Inverse Symbolic Calculator tool, available at http://oldweb.cecm.sfu.ca/projects/ISC/ISCmain.html he obtained (using the "integer relation" option) the result that most likely

$$
\alpha \stackrel{?}{=} \frac{\pi^{2} \log 2}{16} .
$$

The first author then confirmed this detection by using Mathematica to evaluate the right-hand side to over 100 digits, which agreed with a result subsequently obtained for the integral using the "ARPREC" package (which performs arithmetic, together with 1-D, 2-D and 3-D numerical integration, to hundreds or thousands of digits as needed).

Separately, the second author, using Maple Version 10, obtained a numerical value of roughly 20-digit accuracy (actually ten digits suffices), which was then immediately recognized by Maple's "identify" function as the value obtained by the first author's calculations.

In all but a technical sense, we were then done, because we had obtained an analytic evaluation, confirmed numerically to exceedingly high precision. But

\footnotetext{
${ }^{*}$ Lawrence Berkeley National Laboratory, Berkeley, CA 94720, dhbailey@lbl.gov. Supported in part by the Director, Office of Computational and Technology Research, Division of Mathematical, Information, and Computational Sciences of the U.S. Department of Energy, under contract number DE-AC02-05CH11231.

${ }^{\dagger}$ Faculty of Computer Science, Dalhousie University, Halifax, NS, B3H 2W5, Canada, jborwein@cs.dal.ca. Supported in part by NSERC and the Canada Research Chair Programme.
} 
we then were able to establish the result rigorously, by hand or in Maple, by writing $A:=A(\theta, \phi)=1-\sin \theta \cos \phi$, whence the integral can be written

$$
\alpha=\int_{0}^{\pi / 2} \int_{0}^{\pi / 2} \frac{\log (1+A)}{1+A^{2}} \sin \theta d \theta d \phi .
$$

Now by expanding $\log (1+A) /\left(1+A^{2}\right)$ as a double-series and integrating termby-term using

$$
\int_{0}^{\pi / 2} \int_{0}^{\pi / 2} A(\theta, \phi)^{k} \sin \theta d \theta d \phi=\frac{\pi}{2 k+2}
$$

the problem reduces to showing that

$$
\sum_{m=1}^{\infty} \sum_{k=0}^{\infty} \frac{(-1)^{k+m+1}}{(k+1)(2 m+k)}=\frac{\pi}{8} \log 2
$$

Note that (2) is an easy consequence of Wallis' formula and the binomial theorem.

To automate the last step we may use the geometric series twice to write

$$
\begin{aligned}
\sum_{m=1}^{\infty} \sum_{k=0}^{\infty} \frac{(-1)^{k+m+1}}{(k+1)(2 m+k)} & =\int_{0}^{1} \int_{0}^{1} \frac{y}{\left(y^{2}+1\right)(x y+1)} d x d y \\
& =\int_{0}^{1} \frac{\ln (1+y)}{y^{2}+1} d y
\end{aligned}
$$

which last integral, L, Mathematica evaluates directly as $\pi \log 2 / 8$, while Maple gives

$1 / 2 * I * \operatorname{dil} \log (1 / 2+1 / 2 * I)-1 / 2 * I * \operatorname{dil} \log (1 / 2-1 / 2 * I)+1 / 4 * P i * \ln (2)-$ Catalan which simplifies to the desired value via "simplify(convert(value(L),polylog))". Note that the final integrand is precisely the form we exploited in (1).

However, in retrospect, one does not need to employ the intermediate double summation. Instead, one can reason as follows: consider the function $\log (1+$ $A) /\left(1+A^{2}\right)$ expanded in a Taylor series as $c_{0}+c_{1} A+c_{2} A^{2}+\cdots$. Then by using (2) we can rewrite the integral in (1) as

$$
\alpha=\int_{0}^{\pi / 2} \int_{0}^{\pi / 2}\left(\sum_{k=0}^{\infty} c_{k} A^{k}\right) \sin \theta d \theta d \phi=\frac{\pi}{2} \sum_{k=0}^{\infty} \frac{c_{k}}{k+1}
$$

But now we recognize that this series can be seen as the result of evaluating (3) term-by-term:

$$
\int_{0}^{1} \frac{\ln (1+y)}{1+y^{2}} d y=\int_{0}^{1} \sum_{k=0}^{\infty} c_{k} y^{k} d y=\sum_{k=0}^{\infty} \int_{0}^{1} c_{k} y^{k} d y=\sum_{k=0}^{\infty} \frac{c_{k}}{k+1}
$$

and we have the result immediately. 


\section{References}

[1] Prithwijit De, "Problem 11277," American Mathematical Monthly, vol. 114, no. 3 (Mar 2007), pg. 259. 